\title{
PSMA-Based Radioligand Therapy for Metastatic Castration-Resistant Prostate Cancer: The Bad Berka Experience Since 2013
}

\author{
Harshad R. Kulkarni ${ }^{1}$, Aviral Singh ${ }^{1}$, Christiane Schuchardt $^{1}$, Karin Niepsch ${ }^{1}$, Manal Sayeg ${ }^{1}$, Yevgeniy Leshch ${ }^{2}$, \\ Hans-Juergen Wester ${ }^{3}$, and Richard P. Baum ${ }^{1}$ \\ ${ }^{1}$ Theranostics Center for Molecular Radiotherapy and Molecular Imaging, Zentralklinik Bad Berka, Bad Berka, Germany; ${ }^{2}$ Division \\ of Radiopharmacy, Zentralklinik Bad Berka, Bad Berka, Germany; and ${ }^{3}$ Pharmaceutical Radiochemistry, Faculties of Chemistry and \\ Medicine, Technical University Munich, Munich, Germany
}

\begin{abstract}
A potential milestone in personalized nuclear medicine is theranostics of metastatic castration-resistant prostate cancer (mCRPC) based on molecular imaging using PET/CT with ${ }^{68} \mathrm{Ga}$-labeled prostate-specific membrane antigen (PSMA) ligands and molecular radiotherapy using PSMA-targeted radioligand therapy (PRLT) with ${ }^{177}$ Lu-PSMA ligands. ${ }^{68} \mathrm{Ga}-\mathrm{PSMA} \mathrm{PET} / \mathrm{CT}$ enables accurate detection of mCRPC lesions with high diagnostic sensitivity and specificity and provides quantitative and reproducible data that can be used to select patients for PRLT and therapeutic monitoring. Our comprehensive experience over the last 3 years using different radioligands indicates that PRLT is highly effective for the treatment of mCRPC, even in advanced cases, and potentially lends a significant benefit to overall and progression-free survival. Additionally, significant improvement in clinical symptoms and excellent palliation of pain can be achieved.
\end{abstract}

Key Words: PSMA; radioligand therapy; theranostics

J Nucl Med 2016; 57:97S-104S

DOI: 10.2967/jnumed.115.170167

$\mathbf{M}$ etastatic castration-resistant prostate cancer (mCRPC), defined as disease progression despite medical or surgical castration, develops in $10 \%-20 \%$ of prostate cancer patients (1). Most CRPC patients have metastases at diagnosis or develop widespread disease during the first 2 years of follow-up. First-line chemotherapeutic agents such as docetaxel, and second-line chemotherapy with cabazitaxel, are often toxic and prolong life by only a few months (2). Postchemotherapy androgen deprivation therapy with abiraterone acetate and enzalutamide has been shown to prolong overall survival by only 3.9 and 4.8 mo, respectively, compared with placebo. Likewise, the overall survival benefit, compared with placebo, is only 3.6 mo for ${ }^{223}$ Ra-chloride (3-5), which targets only osteoblastic lesions and does not treat nodal or visceral metastases.

PET/CT using ${ }^{68} \mathrm{Ga}$-labeled ligands targeting the prostate-specific membrane antigen (PSMA) is a sensitive diagnostic modality, espe-

\footnotetext{
Received Jul. 3, 2016; revision accepted Aug. 22, 2016.

For correspondence or reprints contact: Richard P. Baum, Theranostics Center for Molecular Radiotherapy and Molecular Imaging, ENETS Center of Excellence, Zentralklinik Bad Berka, 99437 Bad Berka, Germany.

E-mail: richard.baum@zentralklinik.de

COPYRIGHT (C 2016 by the Society of Nuclear Medicine and Molecular Imaging, Inc.
}

cially in poorly differentiated prostate cancer. Its sensitivity in a recent retrospective series by Eiber et al. improved with rising prostatespecific antigen (PSA) velocity, reaching $100 \%$ for a PSA velocity of $5 \mathrm{ng} / \mathrm{mL} / \mathrm{y}$ or more and a Gleason score of 8 or more (6). PSMAtargeted radioligand therapy (PRLT) involves selective binding of a radioligand to PSMA overexpressed in $\mathrm{MCRPC}$ in order to increase tumor dose and spare normal tissue $(7,8)$. Internalization and retention within the tumor cell are essential mechanisms for the cell-killing effect of this radiation therapy (also called endoradiotherapy), which has the advantage of selectively targeting multiple metastases (9).

Theranostics using PET/CT as an in vivo companion diagnostic for decision making and monitoring of radiolabeled peptide therapy or PRLT is an integral part of personalized nuclear medicine $(10,11)$. PSMA-specific PET/CT with ${ }^{68} \mathrm{Ga}$, and PRLT with therapeutic radionuclides such as ${ }^{177} \mathrm{Lu}$, offer a new and unique theranostic possibility by using similar ligands for both diagnosis and therapy. ${ }^{68} \mathrm{Ga}$-PSMA PET/CT is pivotal in selecting mCRPC patients with strong PSMA expression, identifying responders, and personalizing PRLT regimens (12). There is ample evidence of the utility of PET/CT in assessing response, such as with ${ }^{18} \mathrm{~F}$ FDG after radioimmunotherapy of non-Hodgkin lymphoma and with ${ }^{68} \mathrm{Ga}$-somatostatin analogs after peptide receptor radionuclide therapy of neuroendocrine tumors $(13,14)$. Similarly, in PRLT, ${ }^{68} \mathrm{Ga}$-PSMA PET/CT could play an important role in determining prognosis, monitoring therapy, and following up over the long term.

J591 was the first humanized monoclonal antibody targeting the extracellular domain of PSMA (14). Radiolabeled J591 accurately targets bone and soft-tissue prostate cancer metastases for diagnosis or therapy (15). Radioimmunotherapy using ${ }^{177} \mathrm{Lu}-\mathrm{J} 591$ demonstrated antitumor activity and acceptable tolerability in a phase 1 clinical trial (16). To our knowledge, we were the first to use the genetically engineered PSMA minibody IAb2-M-DOTA labeled with ${ }^{111} \mathrm{In}$ for imaging and dosimetry in patients. The ${ }^{111}$ In-labeled compound showed significant uptake in metastatic lesions (17), but hepatic uptake was high when $1 \mathrm{mg}$ was used. Increasing the amount of minibody achieved lower hepatic uptake in accordance with results obtained after administration of the intact J591 parent antibody $(15,17)$.

The research group at Johns Hopkins, in 2002, was the first to implement imaging with a small molecule that targets PSMA (18). The related compounds ${ }^{123}$ I-MIP-1072 and ${ }^{123}$ I-MIP-1095, also bearing a urea-based scaffold, have been used to detect metastatic prostate cancer with SPECT (19). These small molecules demonstrated a potential for therapy when labeled with ${ }^{131} \mathrm{I}(20)$. On the basis of biodistribution and dose calculations for the 
TABLE 1

Current Prerequisites for PRLT

\begin{tabular}{|c|c|}
\hline Prerequisite: the patient... & Details \\
\hline Has cancer of the indicated histologic type & Adenocarcinoma of prostate \\
\hline Meets criterion for CRPC & Testosterone level < 20 ng/dL (22) \\
\hline Has metastatic disease & On ${ }^{68} \mathrm{Ga}-\mathrm{PSMA} \mathrm{PET} / \mathrm{CT}$ \\
\hline \multicolumn{2}{|l|}{ Has tumor progression } \\
\hline PSA progression & According to PCWG2 criteria (23) \\
\hline Radiographic progression & According to RECIST (24) \\
\hline Has not responded to androgen deprivation therapy & LHRH agonists/antagonists, orchidectomy \\
\hline $\begin{array}{l}\text { Has not responded to newer hormonal therapies and } \\
\text { taxane-based chemotherapy }\end{array}$ & Abiraterone, enzalutamide \\
\hline \multicolumn{2}{|l|}{ Has refused taxane-based chemotherapy } \\
\hline \multicolumn{2}{|l|}{ Is medically unfit for chemotherapy } \\
\hline \multicolumn{2}{|l|}{ Has given written informed consent } \\
\hline Has no postrenal obstruction & On ${ }^{99 m T c-M A G 3}$ renal scintigraphy \\
\hline \multicolumn{2}{|l|}{ Has a sufficient... } \\
\hline PSMA expression & On ${ }^{68} \mathrm{Ga}-\mathrm{PSMA}-\mathrm{PET} / \mathrm{CT}$ \\
\hline Life expectancy & $>4-6 \mathrm{mo}$ \\
\hline ECOG performance status & 0 or 1 \\
\hline Karnofsky performance index & $>60 \%$ \\
\hline Hemoglobin level & $\geq 5.5 \mathrm{mmol} / \mathrm{L}^{*}$ \\
\hline White blood cell count & $>3.0 \times 10^{9} / \mathrm{L}$ \\
\hline Platelet count & $>75 \times 10^{9} / \mathrm{L}$ \\
\hline $\begin{array}{l}{ }^{*} \text { Reference range, } 8.6-12.1 \mathrm{mmol} / \mathrm{L} . \\
\text { LHRH = luteinizing hormone-releasing hormone. }\end{array}$ & \\
\hline
\end{tabular}

PSMA-targeted small molecule ${ }^{124}$ I-MIP-1095, the Heidelberg group at the German Cancer Research Center developed a therapy with the analog ${ }^{131}$ I-MIP-1095 that enabled targeted delivery of unprecedented doses to the malignant tissue. The involved lymph node and bone metastases were exposed to mean absorbed doses of more than $300 \mathrm{~Gy}$. However, there was significant toxicity to the salivary glands, with documented xerostomia and mucositis. No renal dysfunction occurred, but thrombocytopenia was noticed (20).
We report here our experience at Zentralklinik Bad Berka since we started, in April 2013, using PRLT with a ${ }^{177}$ Lu-labeled small molecule targeting PSMA.

\section{INDICATION FOR PRLT}

PRLT using ${ }^{177}$ Lu-labeled PSMA ligands is a novel and highly targeted systemic therapy for progressive MCRPC, especially after exhaustion of available conventional therapies such as androgen

TABLE 2

Possible Risk Factors to Consider Before PRLT

\begin{tabular}{|c|c|}
\hline Risk factor & Precaution \\
\hline \multicolumn{2}{|c|}{ Severe bone marrow depression due to... } \\
\hline \multicolumn{2}{|c|}{ Disseminated metastases } \\
\hline \multicolumn{2}{|c|}{ Previous irradiation of spine or pelvis } \\
\hline Concurrent chemotherapy & Allow at least 6 wk before PRLT \\
\hline${ }^{223} \mathrm{Ra}$ therapy & Allow at least $2-3$ mo before PRLT \\
\hline Severe anemia* & Correct by transfusion of packed red blood cells before PRLT \\
\hline \multicolumn{2}{|l|}{ Severe renal insufficiency ${ }^{\dagger}$} \\
\hline Fixed postrenal obstruction & Correct by ureteral stenting or percutaneous nephrostomy before PRLT \\
\hline \multicolumn{2}{|c|}{$\begin{array}{l}{ }^{\star} \text { Hemoglobin level }<5.5 \mathrm{mmol} / \mathrm{L} \text {. } \\
{ }^{\dagger} \text { For example, after chemotherapy }\end{array}$} \\
\hline
\end{tabular}




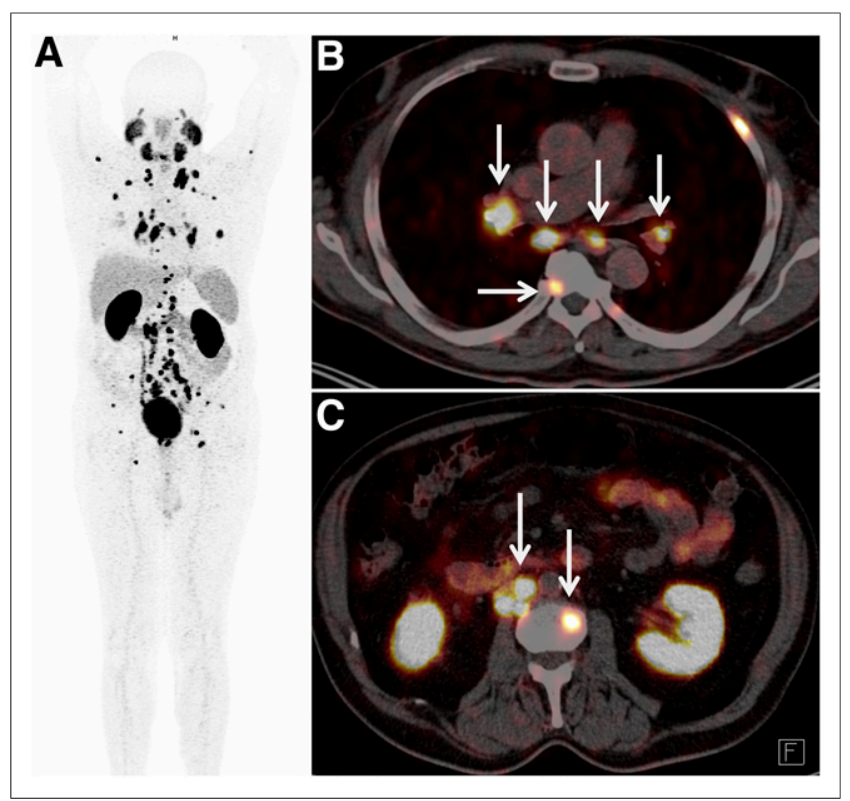

FIGURE 1. First-in-human ${ }^{68} \mathrm{Ga}-\mathrm{SRV} 171 \mathrm{PSMA} P E T / C T$ imaging demonstrating extensive and excellent tracer uptake in soft-tissue (lymph node) and skeletal metastases (arrows). (A) PET maximum-intensityprojection image. (B) Axial PET/CT image of thorax. (C) Axial PET/CT image of abdomen.

deprivation and treatment with abiraterone and enzalutamide, as well as taxane-based chemotherapy (Table 1). Distant metastases with high PSMA expression confirmed on pretherapy ${ }^{68} \mathrm{Ga}$-PSMA $\mathrm{PET} / \mathrm{CT}$, and progressive disease despite extensive previous treatments, are currently essential inclusion criteria as stated in the 2016 consensus recommendations of the German Society of Nuclear Medicine (21). An already decreased bone marrow reserve before PRLT - due to prior chemotherapy or ${ }^{223}$ Ra-therapy of skeletal metastases or to replacement of normal bone marrow by advanced metastatic disease-may predispose to increased bone marrow toxicity (Table 2). PRLT should be performed after correction of anemia by transfusion of red blood cells, which is also imperative to potentiate the tumoricidal effect of molecular radiotherapy by ensuring an oxygen concentration adequate for the formation of free radicals (22).

\section{THE BAD BERKA EXPERIENCE}

\section{Lu-PSMA-TUM1}

${ }^{177}$ Lu-labeled DOTAGA-FFK(Sub-KuE) — developed by the research group at Technical University Munich and given the term ${ }^{177}$ Lu-PSMA-TUM1-led to a significant reduction in metastatic tumor load in $6 \mathrm{mCRPC}$ patients who underwent PRLT using 4.2-8.6 GBq of this compound (23). ${ }^{68} \mathrm{Ga}$-PSMA PET/CT was performed on all patients $1-5 \mathrm{~d}$ before therapy and during followup. High PSMA expression extensively in bone and lymph nodes $(n=4)$, as well as in lung metastases $(n=1)$ and in residual or locally recurrent prostate cancer $(n=2)$, was confirmed by baseline PET/CT and by ${ }^{177} \mathrm{Lu}$-PSMA-TUM1 uptake on posttherapy planar and SPECT/CT images. PSA fell by more than $30 \%$ in 4 of the 6 patients and by more than $50 \%$ in 2 of the 6. Follow-up PET/CT of 3 patients after a single course of PRLT demonstrated partial remission in 2, with an $\mathrm{SUV}_{\max }$ decrease by $51 \%$ and $89 \%$ in the target lesion and disappearance of many previously noted PSMA-positive metastases. There was progression of skeletal metastases in 1 patient. ${ }^{177} \mathrm{Lu}-\mathrm{PSMA}-\mathrm{TUM} 1$ exhibited high tumor uptake, fast renal washout, and rapid blood clearance, resulting in a favorable dosimetry. The following organ and tumor doses were calculated: whole body, $0.02 \pm 0.01 \mathrm{mGy} / \mathrm{MBq}$; kidneys, $0.33 \pm 0.15 \mathrm{mGy} / \mathrm{MBq}$; and tumors, $0.14-5.5 \mathrm{mGy} / \mathrm{MBq}$. Clearance from blood was rapid, with a half-life of up to $42 \mathrm{~h}$. The treatment was tolerated well by all patients, without any significant adverse effects or alterations in any laboratory parameters.

\section{Lu-PSMA-I\&T}

Weineisen et al. focused on further enhancing the affinity and improving the in vivo properties of TUM1 derivatives by increasing the lipophilic interaction of the tracer with PSMA (9). Thus, DOTAGA(I-y)fk(Sub-KuE), given the term ${ }^{177} L u-P S M A-I \& T$ (for imaging and therapy), was developed and evaluated in detail. Our group analyzed the safety and efficacy of ${ }^{177} \mathrm{Lu}-\mathrm{PSMA}-\mathrm{I} \& \mathrm{~T}$ in 56 patients with $\mathrm{mCRPC}$ and found a high objective response rate (7) that resulted in a long progression-free and overall survival (with potential survival benefit) with minimal toxicity.

\section{Lu-PSMA-617}

The small molecule PSMA-617 was demonstrated by recent studies to be safe for PRLT and to have a low toxicity profile, in line with our own experience (24-26).

The different ${ }^{177} \mathrm{Lu}$-labeled ligands (PSMATUM1, PSMA-I\&T, and PSMA-617) used for PRLT yielded similar clinical results, considering efficacy and toxicity profiles.

\section{${ }^{177}$ Lu-PSMA-SRV171}

In 2015 we performed the first ${ }^{68} \mathrm{Ga}$-based PET/CT imaging in patients of mCRPC using a novel PSMA ligand, SRV171 (Fig. 1), which is a potential addition to the existing armamentarium for PRLT when labeled with ${ }^{177} \mathrm{Lu}$.

FIGURE 2. Biodistribution and dosimetry results for normal organs in patients treated with different PSMA radioligands (median uptake in percentage injected activity [\%|A]). (A) Kinetics. (B) Effective half-life in hours. (C) Mean absorbed dose in mGy/MBq $\left(n=38\right.$ for ${ }^{177}$ Lu-PSMA-I\&T and $n=19$ for ${ }^{177}$ Lu-PSMA-617). 
TABLE 3

Absorbed-Dose Constraints and Maximum Activity and Cycles to Reach Them

\begin{tabular}{lccc}
\hline \multicolumn{1}{c}{ Parameter } & Whole body & Kidneys & Parotid glands \\
\hline Absorbed-dose constraint (Gy) & 2 & 23 & 52 \\
\hline Maximum activity to reach constraint (GBq) & & 24 & 47 \\
177Lu-PSMA-I\&T & 79 & 29 & 47 \\
\hline 177Lu-PSMA-617 & 55 & 4 & 8 \\
\hline Possible cycles to reach constraint (6 GBq/cycle) & & 5 & 8 \\
\hline 177Lu-PSMA-I\&T & 13 & 5 & \\
\hline 177Lu-PSMA-617 & 9 & & \\
\hline
\end{tabular}

\section{PRACTICAL PROCEDURE}

The radiopharmaceutical is administered over 5 min with an infusion pump dedicated for radionuclide therapy. In our experience $(>400$ PRLT administrations), the monitored vital parameters (temperature, pulse, and blood pressure) did not significantly change during or after therapy. Hence we suggest that documentation of these vital parameters once before and once after therapy is sufficient. The patients must be well-hydrated, should be encouraged to drink at least 1.5-2 L of fluid, and should receive a 1,000-mL infusion of saline containing $20-40 \mathrm{mg}$ of furosemide after therapy. In contrast to peptide receptor radionuclide therapy with somatostatin analogs, no special nephroprotective measures are required, as the renal absorbed doses were unaffected by whether nephroprotection was used (unpublished data).

\section{DOSIMETRY COMPARISON BETWEEN ${ }^{177}$ LU-PSMA-I\&T AND ${ }^{177}$ LU-PSMA-617}

A comparative intrapatient (i.e., for different PRLT courses) and interpatient dosimetry analysis was performed for ${ }^{177} \mathrm{Lu}$-PSMA-I\&T and ${ }^{177}$ Lu-PSMA-617 (Fig. 2; Table 3). However, a direct head-tohead comparison has some limitations: first, response to therapy and differential tumor load in a particular patient may influence dosimetry after 2 different PRLT courses; second, an interpatient comparison cannot yield completely accurate results, since the kinetics of a certain ligand depend on conditions such as renal function and tumor load. In our experience, the ligands ${ }^{177} \mathrm{Lu}-\mathrm{PSMA}-\mathrm{I} \& \mathrm{~T}$ and ${ }^{177} \mathrm{Lu}-\mathrm{PSMA}-617$ did not significantly differ in response and toxicity profiles.

\section{Whole Body}

We observed a higher whole-body uptake for ${ }^{177} \mathrm{Lu}-\mathrm{PSMA}-617$ at all scan time-points. The time-activity curves demonstrated a biexponential function, that is, an initial rapid decline followed by a second slower decline. The half-lives were shorter for ${ }^{177} \mathrm{Lu}-$ PSMA-I\&T than for ${ }^{177}$ Lu-PSMA-617, which showed a slower washout. The whole-body dose was moderately higher for ${ }^{177} \mathrm{Lu}-$ PSMA-617 because of significantly higher uptake at $20 \mathrm{~h}$ after injection and a longer effective half-life.

\section{Kidneys}

Renal uptake was slightly higher for ${ }^{177} \mathrm{Lu}$-PSMA-I\&T. For both ligands, renal uptake declined rapidly between the first scan and $3 \mathrm{~h}$ after injection, followed by a slower washout with comparable halflives. The resulting renal dose was slightly higher for ${ }^{177} \mathrm{Lu}$-PSMAI\&T than for ${ }^{177}$ Lu-PSMA-617.

\section{Parotid Glands}

In the parotid glands, both ligands demonstrated an initial accumulation of activity until $3 \mathrm{~h}$ after injection followed by an exponential washout. ${ }^{177}$ Lu-PSMA-617 showed higher uptake values and longer half-lives. However, the resulting mean absorbed doses of the different ligands were comparable.

\section{Lacrimal Glands}

Higher uptakes and longer half-lives were observed for ${ }^{177} \mathrm{Lu}-\mathrm{PSMA}-617$, in contrast to ${ }^{177} \mathrm{Lu}-\mathrm{PSMA}-\mathrm{I} \& \mathrm{~T}$, resulting in higher mean absorbed doses to lacrimal glands using ${ }^{177} \mathrm{Lu}-$ PSMA-617.

\section{Red Marrow}

For red marrow, the longest half-life was $94 \mathrm{~h}$; the resulting mean absorbed dose was low, with a mean of $0.03 \mathrm{mGy} / \mathrm{MBq}$ (similar for the 2 ligands). In view of these results concerning the mean absorbed dose to red marrow with a dose limit of $2 \mathrm{~Gy}$, the patients could theoretically be treated using injected activities of up to $200 \mathrm{GBq}$.

\section{Metastases}

We comparatively analyzed 66 metastases (bone, lymph node, liver, lung, and other) in patients treated with ${ }^{177} \mathrm{Lu}$ PSMA-I\&T and 27 metastases (bone and lymph node) in patients treated with

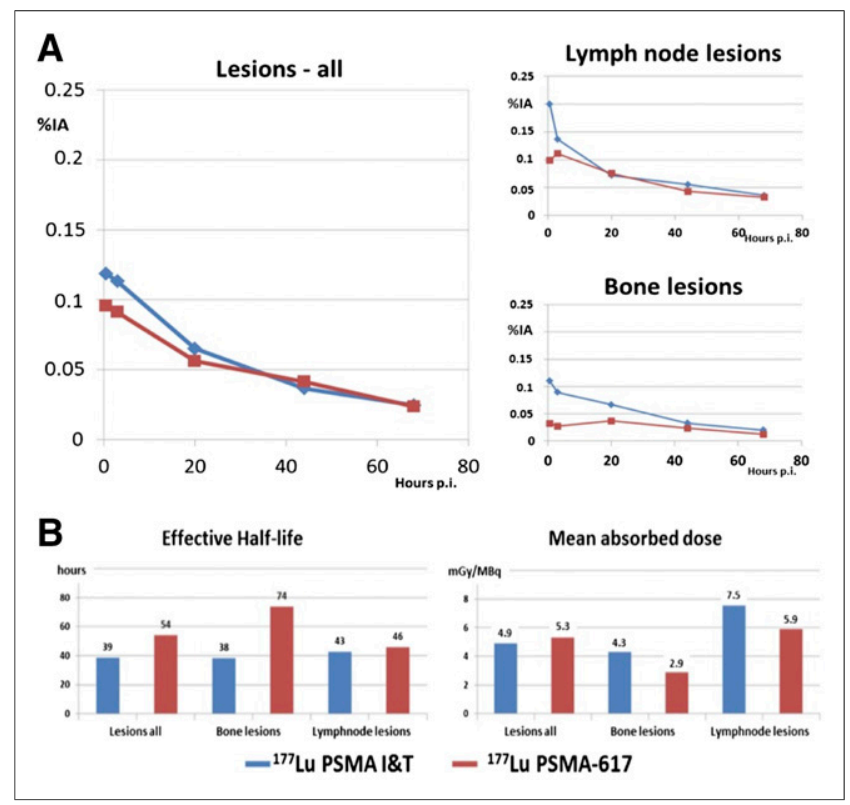

FIGURE 3. (A) Kinetics of tumor lesions and comparative results using ${ }^{177}$ Lu-PSMA-I\&T (blue) and ${ }^{177}$ Lu-PSMA-617 (red) for all lesions, lymph node metastases, and bone metastases. (B) Dosimetric results for different metastases. $\% \mathrm{IA}=$ percentage injected activity; p.i. = after injection. 
TABLE 4

Factors to Consider in Determining Therapeutic Activity and Number of PRLT Cycles

\begin{tabular}{ll}
\hline \multicolumn{1}{c}{ Tumor-related } & Non-tumor-related \\
\hline Gleason score & Age \\
\hline Site of metastatic lesions & Weight \\
\hline Number of metastatic lesions & General condition \\
\hline PSMA expression on tumor cells ${ }^{*}$ & Tumor-related cachexia \\
& Renal function \\
\hline & Single functional kidney \\
\hline & Hematologic status \\
\hline
\end{tabular}

${ }^{*}$ SUV on ${ }^{68} \mathrm{Ga}-\mathrm{PSMA} \mathrm{PET} / \mathrm{CT}$.

${ }^{177}$ Lu-PSMA-617 (Fig. 3). A comparable uptake of both ligands was seen at $20 \mathrm{~h}$ after injection and later, but there were differences early after infusion ( $0.5 \mathrm{~h}$ after injection). ${ }^{177} \mathrm{Lu}$-PSMA-I\&T exhibited a higher initial uptake. After fitting of all time-activity curves to monoexponential functions from $20 \mathrm{~h}$ after injection, a longer half-life was revealed for PSMA-617. The differences in resulting absorbed tumor doses were, however, statistically insignificant. The half-life for ${ }^{177} \mathrm{Lu}-\mathrm{PSMA}-617$ was longer in bone lesions. Despite the relatively shorter half-life of ${ }^{177} \mathrm{Lu}-\mathrm{PSMA}-\mathrm{I} \& \mathrm{~T}$ as compared with ${ }^{177} \mathrm{Lu}-\mathrm{PSMA}-617$, the dose estimations revealed that the highest doses for lymph node lesions occurred when ${ }^{177} \mathrm{Lu}-$ PSMA-I\&T was used. For both ligands, the mean absorbed dose was higher for lymph node metastases than for bone metastases.

\section{PRINCIPLES OF PRLT}

PRLT is based on the principle of personalized medicine, in which number of cycles and amount of administered radioactivity depend on various factors (Table 4). Because bone is the most common site

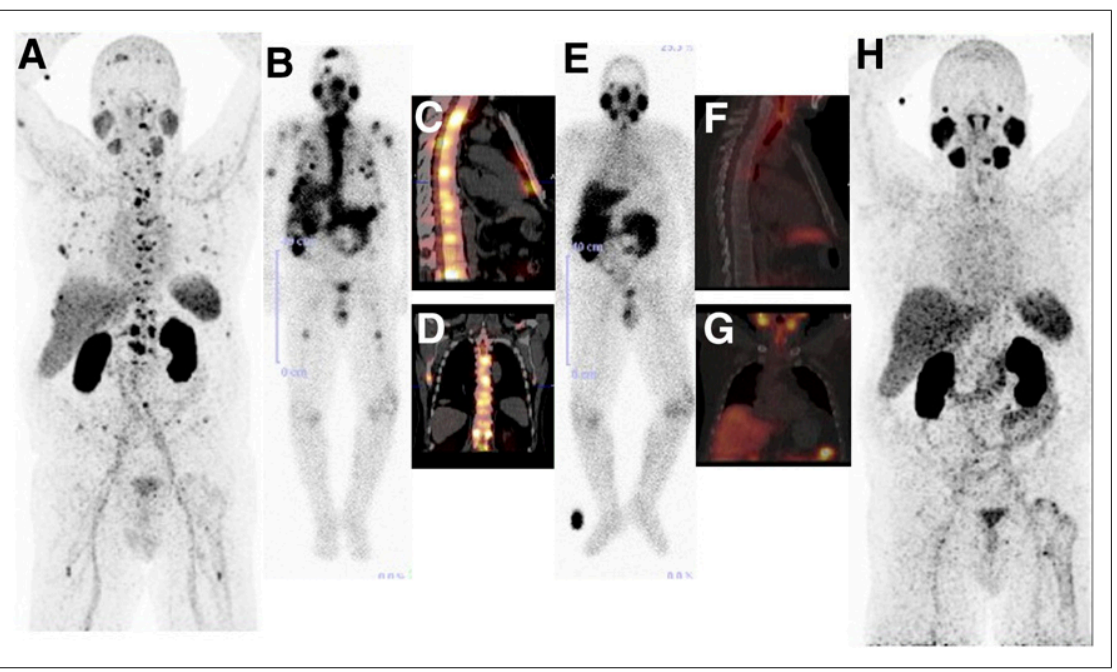

FIGURE 4. Complete remission of disease and 100\% decline in serum PSA, sustained for over 4 mo after PRLT. (A) Numerous ${ }^{68} \mathrm{Ga}$-PSMA-avid skeletal metastases on PET/CT maximum-intensityprojection image before PRLT. (B-D) Excellent uptake on ${ }^{177} \mathrm{Lu}$ images during first treatment $(\mathrm{B}$, whole-body anterior image $20 \mathrm{~h}$ after injection) ( $\mathrm{C}$ and D, SPECT/CT images $41 \mathrm{~h}$ after injection). ( $\mathrm{E}-$ G) Significantly reduced uptake on images after second PRLT 2 mo later $(E$, whole-body anterior image $20 \mathrm{~h}$ after injection) ( $\mathrm{F}$ and $\mathrm{G}, \mathrm{SPECT} / \mathrm{CT}$ images $45 \mathrm{~h}$ after injection). (H) Complete resolution of metastases on ${ }^{68} \mathrm{Ga}-\mathrm{PSMA} \mathrm{PET} / \mathrm{CT}$ image 4 mo after initiation of PRLT. of metastases, a patient with predominantly bone lesions may require application of a relatively higher radioactivity than does a patient with predominantly lymph node lesions. However, although low, the risk of hematotoxicity in a patient with disseminated bone and bone marrow involvement must be kept in mind.

The aim of the first 3 therapy cycles is to achieve remission or stabilization of disease (Fig. 4). Nearly all mCRPC patients who initially respond to PRLT will relapse. In our experience, PRLT can be safely repeated, inducing regression of the disease again (Fig. 5) (27). The maximum tolerable dose of PRLT has not yet been systematically studied. However, frequent therapy cycles (maximum of 8 in our experience so far) over a longer time are feasible for personalized management of the disease. The highest administered activity in a single cycle was $9.7 \mathrm{GBq}$ of ${ }^{177} \mathrm{Lu}-\mathrm{PSMA}$, and this level of activity had no serious adverse effects.

\section{COMPREHENSIVE RESULTS OVER 3 YEARS}

Between April 2013 and April 2016, 119 mCRPC patients (median age, $71 \pm 7 \mathrm{y}$; mean Gleason score, $8 \pm 1$ ) underwent 300 cycles of PRLT. The patients received 1-7 treatments, applying a median activity of $6.0 \mathrm{GBq} /$ cycle (range, 2-9.7 GBq). Bone metastases were present in 96 patients $(80.7 \%)$, whereas $84(70.6 \%)$ presented with lymph node metastases and $19(16.0 \%)$ with visceral metastases. Notably, 24 patients $(20.2 \%)$ had bone metastases only but were not candidates for ${ }^{223} \mathrm{Ra}$ therapy. Lymph node lesions involving more than 2 nodal regions predominated in 19 patients (16.0\%), who had no evidence of skeletal metastases on imaging.

Determination of serum PSA level after at least one course of PRLT in 80 patients revealed a reduction of PSA level in 61 patients $(76.3 \%)$, by more than $50 \%$ in 46 $(57.5 \%)$ and more than $80 \%$ in $22(27.5 \%)$ (Fig. 6A). There was a biochemical complete remission in 2 patients $(2.5 \%)$, who had no detectable PSA after PRLT.

Pain lessened and quality of life improved significantly in symptomatic patients. For example, a dramatic reduction in bone pain was observed in patients who had been wheel 


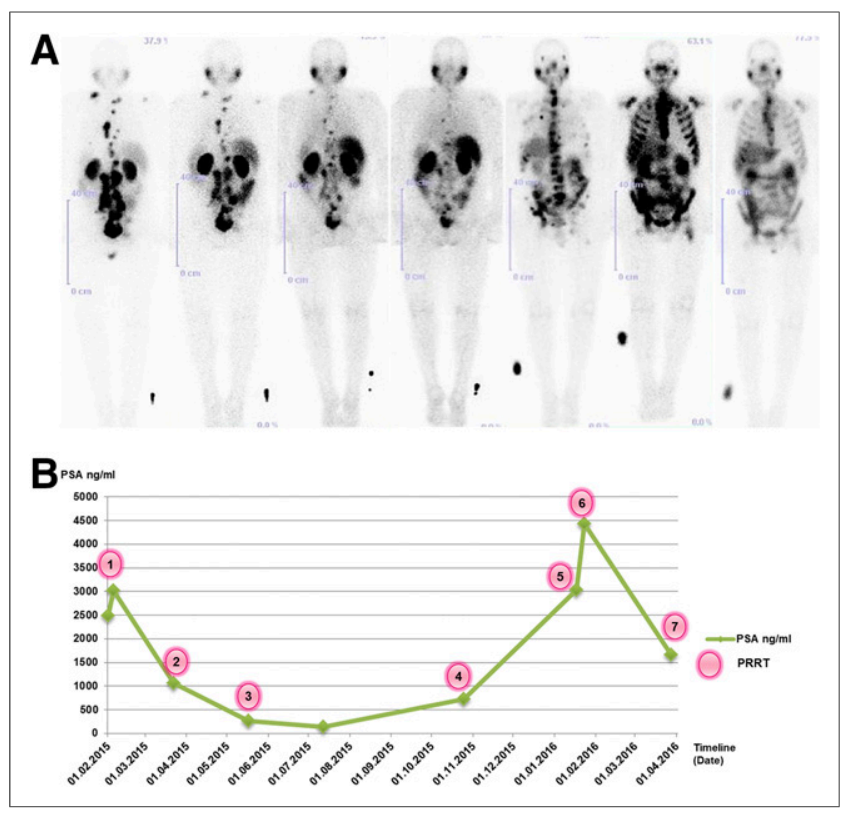

FIGURE 5. (A) Serial ${ }^{177}$ Lu-PSMA whole-body images obtained around $20 \mathrm{~h}$ after injection during (from left to right) first to seventh PRLT cycles. (B) PSA course during the 7 PRLT cycles. Images during first 4 treatments (in posterior view to better demonstrate the findings) show excellent response of multiple lymph nodes and some bone metastases to therapy, whereas images during 5th and 6th treatments (anterior view) show progressive disease with new extensive bone and bone marrow metastases, and image during 7th treatment (anterior view), in March 2016, shows partial remission of bone metastases and significant decrease in uptake that correlates well with significant PSA fall after sixth PRLT cycle, as seen in panel B.

chair-bound before PRLT but could walk without support after PRLT.

In general, the patients tolerated the treatment well and experienced no severe acute or long-term (observation period, $34 \mathrm{mo}$ ) side effects. The most common adverse effect was mild fatigue lasting a few days after therapy. In addition, 5 patients (4.2\%) reported mild dryness of the mouth, most cases of which were reversible. Because of a potentially high radiation dose to the salivary glands, the effect on function can be reproducibly assessed using dynamic salivary gland scintigraphy with ${ }^{99 \mathrm{~m}}$ Tc-pertechnetate before and after PRLT.

Notably, there was no diarrhea despite significant intestinal uptake, in contrast to ${ }^{223} \mathrm{Ra}$ therapy (5). On the contrary, the Heidelberg group reported obstipation as a side effect, which we believe may have been due mainly to the intake of opioid analgesics, as it was not frequently reported in our patient cohort (25). There was no evidence of renal toxicity after PRLT; that is, no significant change was observed in serum creatinine, creatinine clearance (obtained by the CockcroftGault formula), or tubular extraction rate as determined by ${ }^{99 \mathrm{~m}} \mathrm{Tc}-$ mercaptoacetyltriglycine (MAG3) renal scintigraphy. In the initial experience with ${ }^{177}$ Lu-PSMA-I\&T, no grade 3 or 4 hematologic toxicity was found. However, in the extended patient group reported here, 4 patients $(3.4 \%)$ had grade 3 or 4 hematologic toxicity. These patients had been heavily pretreated with chemotherapy or ${ }^{223}$ Ra-therapy of bone metastases and had an already-compromised bone marrow reserve before PRLT. Diffuse bone marrow involvement in combination with previous chemotherapy or ${ }^{223}$ Ra-therapy represents a risk for the development of hematotoxicity. In such cases, therapy with PSMA ligands labeled with $\alpha$-emitters could be beneficial for a highly targeted treatment of metastases (28).
${ }^{68} \mathrm{Ga}$-PSMA PET/CT is a sensitive and specific modality for the detection of both soft-tissue and bone metastases in prostate cancer (29). The response to therapy was assessed using RECIST 1.1 (on contrast-enhanced CT) and molecular response criteria-that is, by determining the change in SUV using the criteria of the European Organization for Research and Treatment of Cancer. ${ }^{68}$ Ga-PSMA PET/CT after at least two courses of PRLT was analyzed in 58 patients (Fig. 6B). The best molecular response (according to the criteria of the European Organization for Research and Treatment of Cancer) was complete remission in 5 patients $(8.6 \%)$. Partial remission was documented in $12(20.7 \%)$, and stable disease in $23(39.7 \%)$. Thus, disease control (reversal of progression [stability or remission of disease]) was achieved in 40 patients $(69.0 \%)$, and mCRPC continued to progress in 18 (31.0\%).

Seventeen patients with extensive metastases $(>20$ bone or lymph node metastases) presented with a PSA level of less than $10 \mathrm{ng} / \mathrm{mL}$, and their PSA value did not correlate with disease burden. This finding may be explained by dedifferentiation of metastases, which therefore manifest with a low serum PSA but a high PSMA-specific uptake. In 6 of these patients, the less than $25 \%$ rise in PSA level was deemed insignificant in light of the stable disease course on PET/CT. On the other hand, 2 patients demonstrated only a mild decrease in serum PSA but a significant reduction in uptake on ${ }^{68} \mathrm{Ga}$-PSMA PET/CT (partial remission based on molecular response criteria); 1 of these 2 patients also

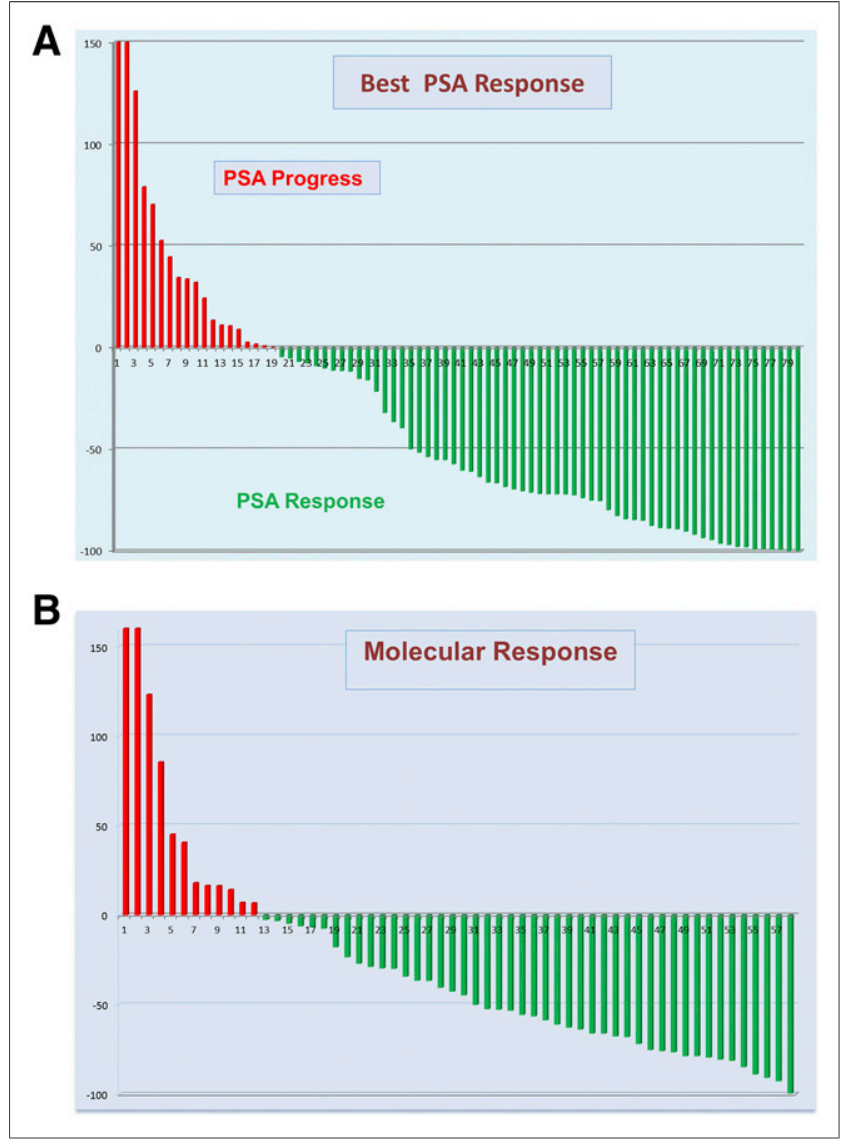

FIGURE 6. (A) Best percentage changes in baseline serum PSA level in 80 patients during follow-up period. (B) Best percentage changes in baseline $\mathrm{SUV}_{\max }$ in 57 patients during follow-up period. 


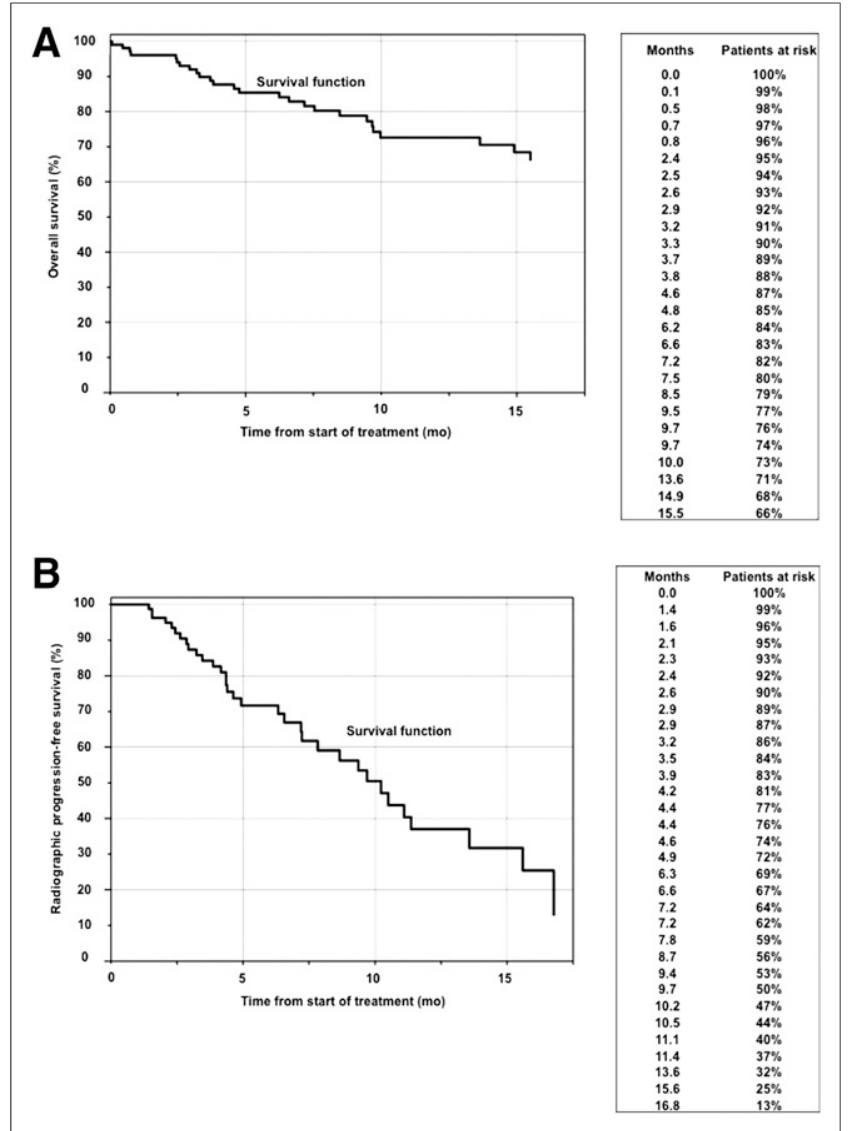

FIGURE 7. Kaplan-Meier curves showing overall (A) and progressionfree (B) survival, according to RECIST 1.1, in 104 patients (observation period, $34 \mathrm{mo})$.

exhibited disease progression based on RECIST 1.1. Both of these patients presented with PSA values of less than $1(0.77$ and $0.05 \mathrm{ng} / \mathrm{mL}$, respectively). In the patient with progressive disease by RECIST, there was less than a 25\% PSA increase; however, this patient also presented with a low PSA level, which did not correspond with the high disease burden.

In general, we found that lymph node metastases of CRPC responded better to PRLT than bone metastases. This finding may be explained by a higher and more uniform radiation dose absorbed by lymph node metastases, which generally exhibit a higher uptake (SUV) on ${ }^{68} \mathrm{Ga}$-PSMA PET/CT than do bone lesions. In addition, the biologic differences in radiation sensitivity might be an influencing factor. ${ }^{68} \mathrm{Ga}$-PSMA PET/CT was superior to $\mathrm{CT}$ alone in assessing the response of skeletal metastases, as the actual size of osteoblastic metastases is difficult to measure and change in size is difficult to appreciate on CT alone. Especially in lymph node metastases, ${ }^{68} \mathrm{Ga}$-PSMA PET/CT detects response at an earlier stage than does CT alone (i.e., molecular response precedes morphologic changes).

In refractory advanced disease, a combination of therapies is often more effective. Six cycles of docetaxel at the beginning of androgen deprivation therapy for $\mathrm{mCRPC}$ resulted in a 13.6-mo longer median overall survival than that with androgen deprivation therapy alone (30). Androgen deprivation therapy may have a synergistic effect with PRLT, as PSMA genes are suppressed by androgens and hence PSMA is upregulated by androgen deprivation in
mCRPC (31). Therefore, we recommend continuation of treatment with luteinizing hormone-releasing hormone analogs during PRLT.

Our patient cohort was quite heterogeneous, having a generally high Gleason score and disease burden-including diffuse bone marrow and visceral involvement—and progressive disease despite extensive pretreatment. Overall, high response rates could be achieved with minimal toxicity despite these negative prognostic factors. The survival data were analyzed in 104 patients (Fig. 7). Over a follow-up period of 34 mo (median, 19 mo), 26 patients died $(25 \%)$. The median overall survival has yet to be reached. Progression-free survival from the commencement of therapy was estimated to be 10.7 mo. The already-compromised bone marrow function due to disseminated bone marrow involvement or prior myelosuppressive therapies (e.g., chemotherapy or ${ }^{223}$ Ra-therapy) limits the application of a higher radioactivity. This limitation might explain a poorer therapeutic response in such patients and the relatively shorter calculated PFS therefore resulting for the entire patient cohort. Potential further treatment cycles could be considered in these patients, taking into account the persistently high PSMA expression of metastases in combination with newer agents such as checkpoint inhibitors (32). The ideal patient for PRLT could possibly be one receiving it before chemotherapy (or maybe in combination with chemotherapy) with good baseline bone marrow function and a good baseline performance status. Therefore, PRLT could potentially be considered at an earlier stage of the disease. The use of PRLT in sequence or in combination with chemotherapy, newer agents (abiraterone, enzalutamide), immune checkpoint inhibitors, and administration of higher radioactivity at shorter intervals should be addressed in future studies.

\section{CONCLUSION}

PRLT using ${ }^{177} \mathrm{Lu}$-labeled PSMA ligands is highly effective for the treatment of mCRPC, even in advanced cases. In addition, significant improvement in clinical symptoms and excellent palliation can be achieved with low or minimal toxicity. Further prospective randomized clinical trials for head-to-head comparison with other treatment modalities are warranted to allow for an unbiased evaluation of survival data and consideration of PRLT earlier in the line of management for mCRPC.

\section{DISCLOSURE}

No potential conflict of interest relevant to this article was reported.

\section{ACKNOWLEDGMENTS}

We would like to express our sincere gratitude to Michael Hofman and Martin Pomper for their critical reading of the manuscript and valuable discussions. We also thank our physician colleagues, the nursing staff, and the nuclear medicine technologists for their support, as well as Sangeeta Ray for the collaboration.

\section{REFERENCES}

1. Kirby M, Hirst C, Crawford ED. Characterising the castration-resistant prostate cancer population: a systematic review. Int J Clin Pract. 2011;65:1180-1192.

2. Basch E, Loblaw DA, Oliver TK, et al. Systemic therapy in men with metastatic castration-resistant prostate cancer: American Society of Clinical Oncology and Cancer Care Ontario clinical practice guideline. J Clin Oncol. 2014;32:3436-3448.

3. de Bono JS, Logothetis CJ, Molina A, et al. Abiraterone and increased survival in metastatic prostate cancer. N Engl J Med. 2011;364:1995-2005. 
4. Scher HI, Fizazi K, Saad F, et al. Increased survival with enzalutamide in prostate cancer after chemotherapy. N Engl J Med. 2012;367:1187-1197.

5. Parker C, Nilsson S, Heinrich D, et al. Alpha emitter radium-223 and survival in metastatic prostate cancer. N Engl J Med. 2013;369:213-223.

6. Eiber M, Maurer T, Souvatzoglou M, et al. Evaluation of hybrid ${ }^{68} \mathrm{Ga}-\mathrm{PSMA}$ ligand PET/CT in 248 patients with biochemical recurrence after radical prostatectomy. J Nucl Med. 2015;56:668-674.

7. Baum RP, Kulkarni HR, Schuchardt C, et al. ${ }^{177} \mathrm{Lu}$-labeled prostate-specific membrane antigen radioligand therapy of metastatic castration-resistant prostate cancer: safety and efficacy. J Nucl Med. 2016;57:1006-1013.

8. Kwekkeboom D. Perspective on ${ }^{177} \mathrm{Lu}$-PSMA therapy for metastatic castrationresistant prostate cancer. J Nucl Med. 2016;57:1002-1003.

9. Weineisen M, Schottelius M, Simecek J, et al. ${ }^{68} \mathrm{Ga}$ - and ${ }^{177} \mathrm{Lu}$-labeled PSMA I\&T: optimization of a PSMA-targeted theranostic concept and first proof-ofconcept human studies. J Nucl Med. 2015;56:1169-1176.

10. Puranik AD, Kulkarni HR, Baum RP. Companion diagnostics. Cancer J. 2015;21:213-217.

11. Baum RP, Kulkarni HR. Theranostics: from molecular imaging using Ga-68 labeled tracers and PET/CT to personalized radionuclide therapy-the Bad Berka experience. Theranostics. 2012;2:437-447.

12. Kulkarni HR, Baum RP. Patient selection for personalized peptide receptor radionuclide therapy using Ga-68 somatostatin receptor PET/CT. PET Clin. 2014;9:83-90.

13. Young H, Baum R, Cremerius U, et al. Measurement of clinical and subclinical tumour response using $\left[{ }^{18} \mathrm{~F}\right]$-fluorodeoxyglucose and positron emission tomography: review and 1999 EORTC recommendations. European Organization for Research and Treatment of Cancer (EORTC) PET Study Group. Eur J Cancer. 1999;35:1773-1782.

14. Kulkarni HR, Baum RP. Theranostics with Ga-68 somatostatin receptor PET/CT: monitoring response to peptide receptor radionuclide therapy. PET Clin. 2014;9:91-97.

15. Bander NH, Trabulsi EJ, Kostakoglu L, et al. Targeting metastatic prostate cancer with radiolabeled monoclonal antibody J591 to the extracellular domain of prostate specific membrane antigen. J Urol. 2003;170:1717-1721.

16. Bander NH, Milowsky MI, Nanus DM, et al. Phase I trial of ${ }^{177}$ lutetium labeled J591, a monoclonal antibody to prostate-specific membrane antigen, in patients with androgen-independent prostate cancer. J Clin Oncol. 2005;23:4591-4601.

17. Schuchardt C, Wiessalla S, Kulkarni HR, et al. Biodistribution and dosimetry of ${ }^{111}$ In PSMA minibody (IAb2-M-DOTA): first results [abstract]. Eur J Nucl Med Mol Imaging. 2013;40(suppl 2):89.

18. Pomper MG, Musachio JL, Zhang J, et al. ${ }^{11} \mathrm{C}-\mathrm{MCG}$ : synthesis, uptake selectivity, and primate PET of a probe for glutamate carboxypeptidase II (NAALADase). Mol Imaging. 2002;1:96-101.
19. Barrett JA, Coleman RE, Goldsmith SJ, et al. First-in-man evaluation of 2 highaffinity PSMA-avid small molecules for imaging prostate cancer. $\mathrm{J}$ Nucl Med. 2013;54:380-387.

20. Zechmann CM, Afshar-Oromieh A, Armor T, et al. Radiation dosimetry and first therapy results with a ${ }^{124} \mathrm{I} /{ }^{131} \mathrm{I}$-labeled small molecule (MIP-1095) targeting PSMA for prostate cancer therapy. Eur J Nucl Med Mol Imaging. 2014;41: 1280-1292.

21. Fendler WP, Kratochwil C, Ahmadzadehfar H, et al. ${ }^{177}$ Lu PSMA-617 therapy, dosimetry and follow-up in patients with metastatic castration-resistant prostate cancer. Nuklearmedizin. 2016;55:123-128.

22. Stanková J, Kavan P, Krízová H, Hermanská E, Dosel P, Sázel M. ${ }^{131}$ I metaiodobenzylguanidine in combination with hyperbaric oxygen therapy in the treatment of prognostically high-risk forms of neuroblastoma [in Czech]. Cas Lek Cesk. 2001;140:13-17.

23. Kulkarni H, Weineisen M, Mueller D, et al. First clinical results with Lu-177 PSMA-TUM1 for the treatment of castrate-resistant metastatic prostate cancer [abstract]. J Nucl Med. 2014;55(suppl 1):10.

24. Ahmadzadehfar H, Eppard E, Kürpig S, et al. Therapeutic response and side effects of repeated radioligand therapy with ${ }^{177} \mathrm{Lu}$-PSMA-DKFZ-617 of castrateresistant metastatic prostate cancer. Oncotarget. 2016;7:12477-12488.

25. Kratochwil C, Giesel FL, Stefanova M, et al. PSMA-targeted radionuclide therapy of metastatic castration-resistant prostate cancer with Lu-177 labeled PSMA-617. J Nucl Med. 2016;57:1170-1176.

26. Rahbar K, Bode A, Weckesser M, et al. Radioligand therapy with ${ }^{177}$ Lu-PSMA617 as a novel therapeutic option in patients with metastatic castration resistant prostate cancer. Clin Nucl Med. 2016;41:522-528.

27. Sabet A, Haslerud T, Pape UF, et al. Outcome and toxicity of salvage therapy with ${ }^{177} \mathrm{Lu}$-octreotate in patients with metastatic gastroenteropancreatic neuroendocrine tumours. Eur J Nucl Med Mol Imaging. 2014;41:205-210.

28. Kratochwil C, Bruchertseifer F, Giesel FL, et al. ${ }^{225}$ Ac-PSMA-617 for PSMA targeting alpha-radiation therapy of patients with metastatic castration-resistant prostate cancer. J Nucl Med. July 7, 2016 [Epub ahead of print].

29. Afshar-Oromieh A, Avtzi E, Giesel FL, et al. The diagnostic value of PET/CT imaging with the ${ }^{68} \mathrm{Ga}$-labelled PSMA ligand HBED-CC in the diagnosis of recurrent prostate cancer. Eur J Nucl Med Mol Imaging. 2015;42:197-209.

30. Sweeney CJ, Chen Y, Carducci M. Chemohormonal therapy in metastatic hormone-sensitive prostate cancer. N Engl J Med. 2015;373:737-746.

31. Wright GL Jr, Grob BM, Haley C, et al. Upregulation of prostate-specific membrane antigen after androgen-deprivation therapy. Urology. 1996;48: 326-334.

32. Postow MA, Callahan MK, Wolchok JD. Immune checkpoint blockade in cancer therapy. J Clin Oncol. 2015;33:1974-1982. 